\title{
State-Space Estimation for OFDM and SC-FDE Schemes with Strongly Varying Carrier Frequency Offset
}

\author{
Pedro Pedrosa ${ }^{1}$, Rui Dinis ${ }^{1}$, and Fernando Nunes ${ }^{2}$, Non-members
}

\begin{abstract}
Parametric algorithms for the estimation of rapidly-varying Carrier Frequency Offset (CFO) usually employ pilot symbols multiplexed with the data transmission. As the $\mathrm{CFO}$ variation rate increases so has to increase the density of pilot symbols transmitted, thus impairing the bandwidth efficiency. In order to reduce the number of pilot symbols used in the estimation of rapidly-varying $\mathrm{CFO}$ it was proposed to use a truncated Taylors series to predict the CFO, where the derivatives up to order $d-1$ are recursively estimated with a d-order Kalman filter (KF). We propose to compare the performance of a fourth-order $\mathrm{KF}$ predictor in the most popular block transmission systems: Orthogonal Frequency Division Multiplexing (OFDM) and Single Carrier Frequency-Domain Equalization (SC-FDE).

Simulating different transmission scenarios, e.g., channel coding and spatial diversity, our results show that for static multipath fading channels the proposed receiver for the SC-FDE scheme exhibits better Bit Error Rate (BER) performance than that of OFDM.
\end{abstract}

Keywords: Carrier frequency offset, Kalman filter, orthogonal frequency division multiplexing, single carrier frequency-domain equalization.

\section{INTRODUCTION}

Future wireless systems will support ever increasing data rates. Presently Orthogonal Frequency Division Multiplexing (OFDM) and Single Carrier Frequency-Domain Equalization (SC-FDE) transmission schemes are presented as valid solutions to support future applications. OFDM and SC-FDE can cope with highly dispersive channels without requiring complex receiver structures $[1,2]$. In order to prevent Inter-Symbol Interference (ISI), which occurs in multipath channels, a guard interval is added by extending the signal with a Cyclic Prefix (CP). This guard interval has to be larger than the overall Channel Impulse Response (CIR) and, in order to maintain bandwidth efficiency, it should be a small

\footnotetext{
${ }^{1}$ The authors are with the ISR Instituto Superior Tcnico, 1049-001 Lisboa, Portugal. E-mail:ppedrosa@isr.ist.utl.pt, rdinis@isr.ist.utl.pt

${ }^{2}$ The authors are with the IT Instituto Superior Tcnico, 1049001 Lisboa, Portugal. E-mail:nunes@lx.it.pt
}

fraction of the total symbol duration. This leads to longer symbols intervals which the characteristics of the channelare almost certain to vary. And, as frequency errors can not exceed a small fraction of the inverse of the total symbol duration, we have that block transmission is very sensitive to Carrier Frequency Offset $(\mathrm{CFO})$. One source of $\mathrm{CFO}$ is the frequency mismatch between the oscillators at the transmitter and receiver. Another possible source of CFO is the Doppler frequency shift caused by relative motion between the transmitter and the receiver. CFO estimation becomes more challenging in a Time Division Multiple Access (TDMA) scheme where the non-continuity of the users time-slots makes the use of previous estimates less helpful. There are two basic approaches for CFO estimation (see [3] and references within): using pilot symbols to extract the carrier frequency and phase of the received signal; or deriving the carrier frequency directly from the modulated signal, usually called a blind scheme. The former option will always require additional bandwidth consumption. Alternatively, in SC-FDE schemes, differentially encoded transmission proved to be robust to the presence of CFO.

There are various data-aided CFO estimators described in the literature for OFDM schemes. In [4] Moose proposed a Maximum-Likelihood (ML) CFO estimator, based on the use of two identical and consecutive symbols, with a frequency acquisition range $\pm 1 /(2 T)$, where $T$ is the "useful" symbol duration. This result was later extended in [5]. Morelli and Mengalli proposed in [6] a estimator based on the Best Linear Unbiased Estimator (BLUE) principle with the use of only one pilot symbol having $L>2$ identical parts and allowing a frequency aquisition range of $\pm L /(2 T)$. When dealing with rapidly-varying CFO, a situation likely to occur in mobile telecommunications, the estimates are obtained at the pilot symbols and predicted at the data symbols. In [7], a statespace solution was devised, displaying overall superior results to those presented by conventional linear algorithms.

In this article we propose comparing the OFDM system with the SC-FDE system when resorting to a state-space estimation of rapidly-varying CFO. Since channel coding is important in OFDM systems [8], leading to a very high coding gain, especially if soft decision decoding is applied, we compare both OFDM 
and SC-FDE systems under these conditions.

This paper is organized as follows: in sec. 1 we introduce this paper; in sec. 2 we characterize the proposed systems; in sec. 3 we present the statespace solution for the CFO estimation problem; in sec. 4 we describe the structure of the proposed receivers for OFDM and SC-FDE schemes; in sec. 5 we analyze the obtained performance results; and sec. 6 concludes this paper.

\section{SYSTEM CHARACTERIZATION}

In Fig. 1 we sketch the basic OFDM transmission scheme. The information bits to be transmitted are mapped into a constellation, e.g., QPSK, according to a valid rule, e.g., Gray coding. The resulting frequency-domain signal is converted to timedomain through the Inverse Discrete Fourier Transform (IDFT) operation, effectively performed by the Inverse Fast Fourier Transform (IFFT) algorithm. We add a CP to the time domain signal and send it through the channel. In the receiver, after the $\mathrm{CP}$ removal, the time-domain signal is converted to frequency-domain through the Discrete Fourier Transform (DFT) operation performed by the Fast Fourier Transform (FFT) algorithm. We proceed with the frequency-domain equalization and, after the corresponding demapping, we obtain the received data bits.

The block diagram of the basic SC-FDE transmission scheme is depicted in Fig. 2. Comparing Fig. 2 with Fig. 1, we can see that both OFDM and SCFDE systems are very similar. The blocks required for its implementation are the same; the main difference being that for the SC-FDE case the IDFT block is found not in the transmitter side but on the receiver. So, the SC-FDE scheme and the OFDM scheme presents the same complexity [2].

Let $p \geq 2$ be the period of the pilot symbols in symbol intervals. The CFO has to be predicted at the data symbols using the estimates obtained at the pilot symbols. In [7], a state-space approach was proposed whereby the CFO is modeled as the first component of a d-dimensional state vector which is recursively estimated by a Kalman Filter $(\mathrm{KF})$. Let $v(t)=f_{0} T$ stand for the (symbol duration) normalized CFO. Using the truncated Taylor series we approximate $v$ at $t+\tau$, with $\tau>0$, by

$v(t+\tau) \approx v(t)+\tau(t)+\left(\tau^{2} / 2\right) \ddot{v}(t)+\ldots$

$$
+\left[\tau^{d-1} /(d-1) !\right] v^{d-1}(t) .
$$

and resort to the KF to estimate $v(t)$ and the derivatives $(t),(t), \ldots$.

Consider the time-domain size- $\mathrm{N}$ block of received data symbols $\left\{y_{n} ; n=0,1, \ldots, N-1\right\}$ in the presence of $\mathrm{CFO}$

$$
\dot{y}_{n}=\exp \left[j\left(\frac{2 \pi n v}{N}+\beta_{0}\right)\right] \omega_{n}+n_{n} .
$$

with $v$ being normalized (to the symbol duration $T$ ) time-varying CFO and $\beta_{0}$ is the unknown origin phase which can be coped with through pilot symbols or differential encoding techniques. The quantity $n_{n}$ is zero mean gaussian noise with variance $\sigma_{n}^{2}=E\left[\left|n_{n}\right|^{2}\right]$, and

$$
\omega_{n}=\operatorname{IDFT}\left\{S_{k} H_{k}\right\} .
$$

where $H_{k}$ is the channel frequency response, and IDFT $\{$.$\} means the Inverse Discrete Fourier Trans-$ form operation. In (3), for OFDM systems, $\left\{S_{k} ; k=0,1, \ldots, N-1\right\}$ are the frequency domain data symbols chosen from a given constellation, e.g., QPSK, and, for SC systems, $\left\{S_{k} ; k=0,1, \ldots, N-1\right\}$ $=\operatorname{DFT}\left\{s_{n} ; n=0,1, \ldots, N-1\right\}$, where $\left\{s_{n} ; n=\right.$ $0,1, \ldots, N-1$ are the time domain data symbols chosen from a given constellation, and DFT $\{$.$\} means the$ Discrete Fourier Transform operation. The Signal-toNoise Ratio is defined as $\operatorname{SNR}=\sigma_{\omega}^{2} / \sigma_{n}^{2}$ where $\sigma_{n}^{2}=$ $\mathrm{E}\left[\left|n_{n}\right|^{2}\right]$.

As in [6], the pilot symbols consist of a set of pseudo-random values $\left\{S_{k} ; k=0,1, \ldots, N-1\right\}$ with $\left|S_{k}\right|=\sqrt{L}$ for $(k \bmod L)=0$, and $\left|S_{k}\right|=0$ for $(k$ $\bmod L) \neq 0$, where $L=2,4,8, \ldots$., and $(i \bmod M)$ stands for the integer remainder of the division of $i$ by $M$. For the data symbols we admit, with no loss of generality, that $\left\{\left|S_{k}\right|=1 ; k=0,1, \ldots, N-1\right\}$.

The samples $y_{n, l}$ are separated into two streams: the pilot symbols, corresponding to $(1 \bmod p)=0$ and the data symbols, corresponding to $(1 \bmod p)=1, \ldots$. . , $p-1$, for the lth received burst. The arrangement of the pilot symbols is sketched in Fig. 3 .

The pilot symbols are split into $L$ sections of $M_{p}$ samples, $\left[y_{(m-1) M_{p}}, \ldots, y_{m M p-1}\right], m=1, \ldots, L$, where we drop the burst index 1 for simplification.

Consider now the correlation between subsets $\left[y_{0}, \ldots, y_{N-m M_{p}-1}\right]$ and $\left[y_{m M_{p}}, \ldots, y_{N-1}\right]$ as

$$
\begin{gathered}
R(m)=\frac{1}{N-m M_{p}} \sum_{n=m M_{p}}^{N-1} y_{n} y_{n-m M_{p}}^{*}, \\
m=1, \ldots, L-1 .
\end{gathered}
$$

The case $2 m=L$ corresponds to the Schmidl and Cox algorithm.

For $n_{n}=0$ it can be easily shown that

$$
v=\frac{L}{2 \pi m} \arg \{R(m)\}
$$

In the presence of weak noise we can write

$$
R(m)=D \exp \left(j \frac{2 \pi n v}{L}\right)[1+\gamma(m)] .
$$


where $D$ is a real quantity depending on the channel response and $\gamma(m)$ is a complex quantity that depends on the channel noise, such that $|\gamma(m)|\langle<1$, with high probability [6]. Thus, a normalized CFO estimate can be obtained as

$$
\tilde{v}=\frac{L}{2 \pi m} \arg \{R(m)\}=v+\frac{L}{2 \pi m} \gamma_{I}(m)
$$

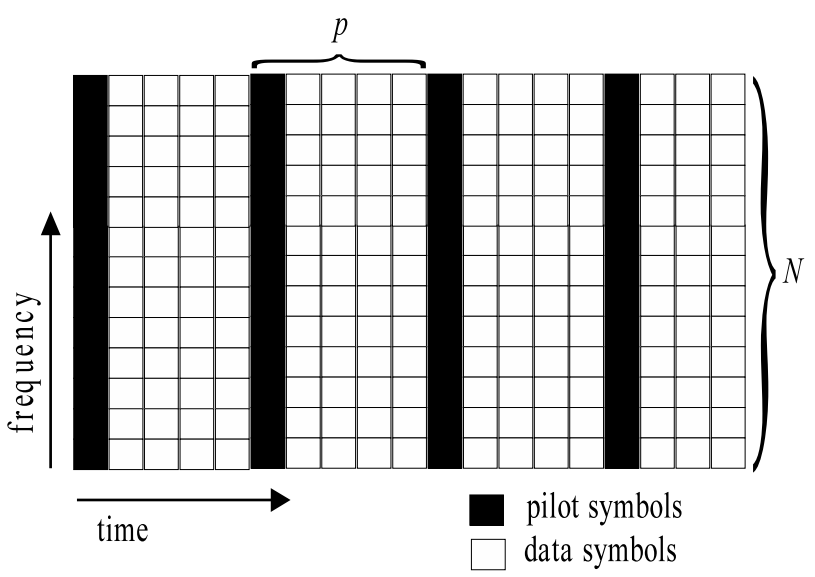

Fig.3: Arrangement of the pilot symbols.

$\gamma_{I}(m)$ is the imaginary part of $\gamma(m)$. Simulations have shown that for $N 1, \gamma_{I}(m)$ is approximately gaussian. The acquisition range of the estimator (7) is

$$
|\tilde{v}| \leq L / 2 m \text {. }
$$

For $L$ constant, the range depends on $m$, and is maximized for $m=1$ with $|\tilde{v}| \leq L / 2$.

Reference [6] describes how to estimate $v$ based on the BLUE principle. However, for rapidly-varying CFOs the percentage of pilot symbols has to be high, thus impairing the bandwidth efficiency. In the next section we propose to use the estimates (7) as the input of a KF that filters $\tilde{v}$ at the pilot symbols and predicts $\hat{v}$ at the data symbols for the OFDM and SC-FDE schemes.

\section{STATE-SPACE SOLUTION}

Assume that the CFO can be well extrapolated, at the data symbols, using the Taylor series in (1) with terms up to the third derivative, i.e., $d=4$. Let the continuous-time state vector $\mathrm{X}(t)=\left[\mathrm{x}_{1}(t) \ldots \mathrm{x}_{4}(t)^{\mathrm{T}}\right]$, with $x_{1}(t)=v(t)$ and $x_{g}=v^{(g-1)}(t), g=2, \ldots, d$. The dynamics equation is

$$
\left[\begin{array}{l}
\dot{x}_{1}(t) \\
\dot{x}_{2}(t) \\
\dot{x}_{3}(t) \\
\dot{x}_{4}(t)
\end{array}\right]=\underbrace{\left[\begin{array}{llll}
0 & 1 & 0 & 0 \\
0 & 0 & 1 & 0 \\
0 & 0 & 0 & 1 \\
0 & 0 & 0 & 0
\end{array}\right]}_{F}\left[\begin{array}{l}
x_{1}(t) \\
x_{2}(t) \\
x_{3}(t) \\
x_{4}(t)
\end{array}\right]+\underbrace{\left[\begin{array}{l}
0 \\
0 \\
0 \\
1
\end{array}\right]}_{\Gamma} w(t)
$$

where $w(t)$ is zero mean gaussian noise with power spectral density $G_{W}(f)=q_{c}$.

The discrete-time state vector for a discretization interval $\Delta=p$, at the pilot symbol $i$, is $X_{i}(t)=$ $\left[\mathrm{x}_{1, i}(t) \mathrm{x}_{4, i}(t)\right]^{T}$.

The corresponding dynamics equation is

$$
X_{i+p}=\Phi(\Delta) X_{i}+\Gamma w_{i}, \quad i=0, p, 2 p, \ldots
$$

where the state transition matrix is

$$
\Phi(\Delta)=e^{F \Delta}=\left[\begin{array}{cccc}
1 & \Delta & \Delta^{2} / 2 & \Delta^{3} / 6 \\
0 & 1 & \Delta & \Delta^{2} / 2 \\
0 & 0 & 1 & \Delta \\
0 & 0 & 0 & 1
\end{array}\right]
$$

and $w_{i}$ is a zero mean white gaussian sequence with variance $q=q_{c} \Delta$.

The observations model is $z_{i}=\tilde{v}_{i}$ with

$$
z_{i}=\underbrace{\left[\begin{array}{llll}
1 & 0 & 0 & 0
\end{array}\right]}_{s} X_{i}+v_{i}
$$

where $v_{i}$ is a zero-mean white gaussian sequence uncorrelated with $w_{i}$ and with variance $r$, that quantifies the effect of noise in (7). The KF associated with (9)-(12) estimates recursively the state vector $X_{i}$. The following equations are used, with $\hat{X}(i \mid i-p)$ and $\hat{X}(i \mid i)$ denoting respectively the $p$-step prediction and filtering estimates at iteration $i[8]$.

-prediction step

$$
\begin{gathered}
\hat{X}(i \mid i-p)=\Phi(\Delta) \hat{X}(i-p \mid i-p) ; \\
\mathrm{P}(i \mid i-p)=\Phi(\Delta) \mathrm{P}(i-p \mid i-p) \Phi^{T}(\Delta)+q \Gamma \Gamma^{T} .
\end{gathered}
$$

where $(P(i \mid i-p)$ and $P(i \mid i)$ are respectively the $p$ step prediction and filtering covariance matrices at iterationi.

$$
\begin{aligned}
& \text { •filtering step } \\
& \qquad \begin{array}{l}
\hat{\mathrm{X}}(i \mid i)=\hat{\mathrm{X}}(i \mid i-p)+G_{i}\left[z_{i}-S \hat{\mathrm{X}}(i \mid i-p)\right] \\
P(i \mid i)=P(i \mid i-p)-G_{i} S P(i \mid i-p)
\end{array}
\end{aligned}
$$

where $G_{i}=P(i \mid i-p) S^{T}\left[S P(i \mid i-p) S^{T}+r\right]^{-1}$ is the Kalman gain with $S$ being defined in (12).

The KF is initialized at iteration $i=0$ by assigning values to $\hat{\mathrm{X}}(0 \mid-p)$ and $P(0 \mid-p)$

At the pilot symbol $i$ the $\mathrm{CFO}$ is estimated as $\hat{v}_{i}=S \hat{\mathrm{x}}(i \mid i)$. At the data symbol $i+h, h$ 


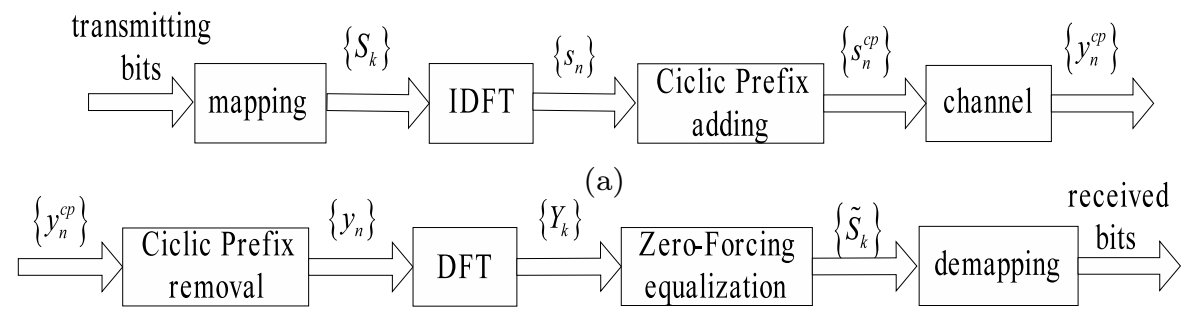

(b)

Fig.1: Basic OFDM scheme:(a) transmitter and channel; (b) receiver.

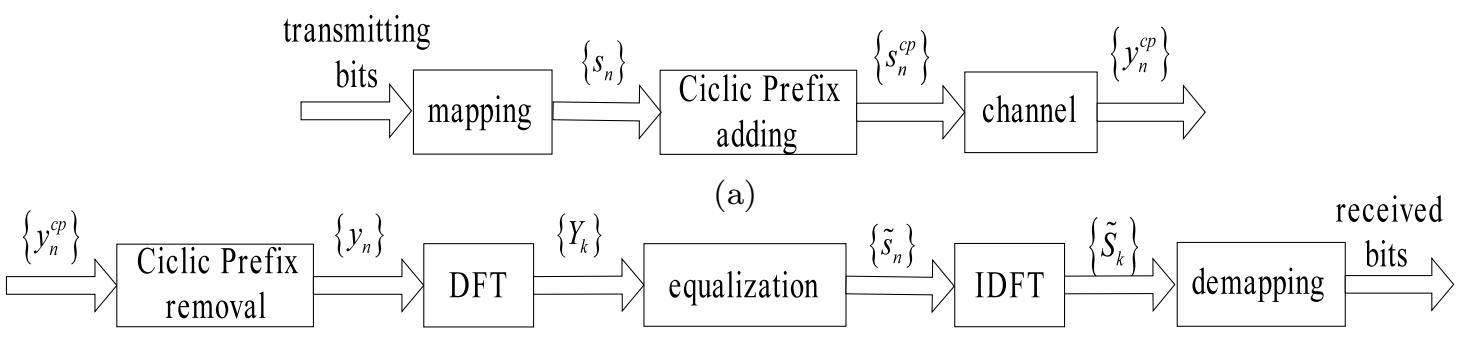

(b)

Fig.2: Basic SC-FDE scheme:(a) transmitter and channel; (b) receiver.

$=1, \ldots, p-1$, the $\mathrm{CFO}$ is estimated (predicted) as $(i+h)=S(i+h \mid i)=S \Phi(h)(i \mid i)$. Using (10)- (12), yields

$$
\begin{array}{r}
\hat{v}(i+h)=x_{1, i}+h x_{2, i}+\left(h^{2} / 2\right) \hat{x}_{3, i}+ \\
\left(h^{3} / 6\right) \hat{x}_{4, i}, h=o, \ldots, p-1
\end{array}
$$

Equation (15) is compatible with (1) provided that $\tau=h$ and $\hat{v}^{(a)}(t)=\hat{x}_{a+1, i}, a=0, \ldots 3$.

The computational effort required by the $\mathrm{KF}$ is small and can be further reduced by noting that the propagation of the covariances matrices, $P(i \mid i-p)$ and $P(i \mid i)$, is independent of the input data allowing its computation to be carried out off-line. In addition, a slightly suboptimal algorithm can be used where $P(i \mid i-p)$ and $P(i \mid i)$ are replaced with their steadystate $(i \rightarrow \infty)$ counterparts.

\section{RECEIVERS}

The proposed receiver schemes are sketched in Fig. 4 where perfect symbol synchronization is assumed. The received time domain samples $\left\{\dot{y}_{n, 1} ; n=0,1, \ldots, N-1\right\}(1=1,2, \ldots)$ are separated into pilot and data stream which are processed separately. The processing block of the pilot symbols consists of a CFO estimator corresponding to (4) and (7). The CFO prediction for $(1 \bmod p) \neq 0$ is accomplished by the KF. The samples of the data stream are phase de-rotated according to

$$
y_{n, l}=\dot{y}_{n, l} \exp \left(-j 2 \pi \frac{n \hat{v} l}{N}\right) .
$$

The DFT is then applied to produce the frequencydomain samples $\left\{Y_{k}^{(j)} ; k=0,1, \ldots, N-1\right\}$ where we drop the 1 index term, for simplification, and introduce the index for the $j$ th diversity branch, admitting a diversity order $J$. For the OFDM scheme we use a zero-forcing equalization procedure that yields

$$
\tilde{S}_{k}=\frac{\sum_{j=1}^{J} Y_{k}^{(j)}\left(H_{k}^{(j)}\right)^{*}}{\sum_{j=1}^{J}\left|H_{k}^{(j)}\right|^{2}} .
$$

The SC-FDE receiver employs a joint carrier synchronization and equalization procedure which combines IB-DFE (Iterative Block Decision Feedback Equalization) and a post-equalization carrier synchronization scheme as presented in [9]. Within each iteration of the equalizer we perform a decision-directed frequency offset estimation as depicted in Fig. 5. For a given iteration $i$, the frequency-domain samples at the output of the IB-DFE are given by

$$
\tilde{S}_{k}^{(i)}=\sum_{j=1}^{j} F_{k}^{(j, i)} Y_{k}^{(j)}-B_{k}^{(i)} \hat{S}_{k}^{i-1} .
$$

where $\left\{F_{k}^{(j, i)} ; k=0,1, \ldots, N-1\right\}(j=1,2, \ldots, j)$ and $\left\{B_{k} ; k=0,1, \ldots, N-1\right\}$ represent, respectively, the 
feedforward and feedback coefficients. $\left\{\hat{S}_{k}^{(i-1)} ; k=0,1\right.$ . . . , N - 1represents the DFT of the data estimates $\left\{\hat{S}_{n}^{(i-1)} ; n=0,1, \ldots, N-1\right\}$ that are obtained from the time-domain samples present at the equalizer output in the previous iteration $\left\{\tilde{S}_{n}^{(i-1)} ; n=0,1, \ldots, N-1\right\}=$ IDFT $\left\{\tilde{S}_{k}^{(i-1)} ; k=0,1, \ldots, N-1\right\}$. The optimum values for the feedforward coefficients $F_{k}^{(j, i)}$ are given by [10]

$$
\begin{gathered}
F_{k}^{(j, i)}=\frac{\left(H_{k}^{(j)}\right)^{*}}{\alpha^{-1}+\left[1-\left(\rho^{(i-1)}\right)\right]^{2} \sum_{j=1}^{J}\left|H_{k}^{(j)}\right|^{2}} \\
j=1,2, \ldots, J .
\end{gathered}
$$

where $\alpha=E\left[S_{k}\right] / E\left[N_{k}\right]$ with $\left\{N_{k} ; k=0,1, \ldots, N-1\right\}$ $=\operatorname{DFTf}\left\{n_{n} ; n=0,1, \ldots, N-1\right\}$ and the correlation coefficient $\rho^{(i)}$ is defined as

$$
\rho^{(i)}=\frac{E\left[S_{n}\left(\hat{S}_{n}^{(i)}\right)^{*}\right]}{E_{s}} .
$$

with $E_{S}=E\left[\left|S_{n}\right|^{2}\right]$ representing the mean symbol energy. Notice that, for the first iteration $(i=0)$, there is no information about $S_{k}$ and the correlation is zero, corresponding to linear FDE.

The optimum values for $B_{k}^{(i)}$ are given by

$$
B_{k}^{(i)}=\rho^{(i-1)}\left(\sum_{j=1}^{J} F_{k}^{i, j} H_{k}^{(j)}-\gamma^{(i)}\right) .
$$

The quantity $\gamma^{(i)}$ can be seen as the global average of the channel frequency response after the $J$ feedforward filter outputs are combined, i.e.,

$$
\gamma^{(i)}=\frac{1}{N} \sum_{k=0}^{N-1} \sum_{j=0}^{J} F_{k}^{(j, i)} H_{k}^{(j, i)}
$$

The received symbol estimates are obtained through hard-decisions. For the OFDM scheme this is done in frequency-domain

$$
\hat{S}_{k}=\operatorname{HD}\left(\tilde{S}_{k}\right)
$$

where $\mathrm{HD}()=.\operatorname{sign}(\Re\{\})+.j \operatorname{sign}(\Im\{\}$.$) is the hard-$ decisions operation. For SC-FDE scheme the harddecisions are done in time-domain

$$
\hat{s}_{n}=\mathrm{HD}\left(\tilde{s}_{n}\right)
$$

where $\left\{\tilde{s}_{n} ; n=0,1, \ldots, N-1\right\}=\operatorname{IDFTf} \tilde{S}_{k} ; k=$ $0,1, \ldots, N-1$.

We can define the remaining offset present in $y_{n}$ after the first CFO compensation (16) as being

$$
\delta_{V}=v-\hat{v}
$$

For the SC-FDE receiver we resort to a decisiondirected $\mathrm{CFO}$ estimator in order to obtain a $\delta \mathrm{v}$ estimate

$$
\begin{gathered}
\hat{\delta}_{V}=\frac{N}{2 \pi M_{d} T} \arg \{\xi\} . \\
\xi=\sum_{n=0}^{N-M_{d}-1} \frac{y_{n}+M_{d} y_{n}^{*}}{s_{n}+M_{d} s_{n}^{*}} .
\end{gathered}
$$

for $M_{d}>N / 2$.

The post-equalization phase rotation embedded in the IB-DFE is given by

$$
\hat{s}_{n}^{(i)}=\hat{\hat{S}}_{n}^{(i)} \exp \left(-j 2 \pi \frac{n \hat{\delta} V}{N}\right) .
$$

\section{PERFORMANCE RESULTS}

In order to compare the performance of the SCFDE and the OFDM schemes we consider QPSK modulated bursts with $N=1024$ symbols transmitted over a strongly frequency-selective Rayleigh fading channel. We adopt the power delay profile type C within HIPERLAN/2, with uncorrelated Rayleigh fading on the different paths. An ideal coherent detection, with perfect synchronization and channel estimation, is assumed.

For simulation purposes, the normalized $\mathrm{CFO}$ is

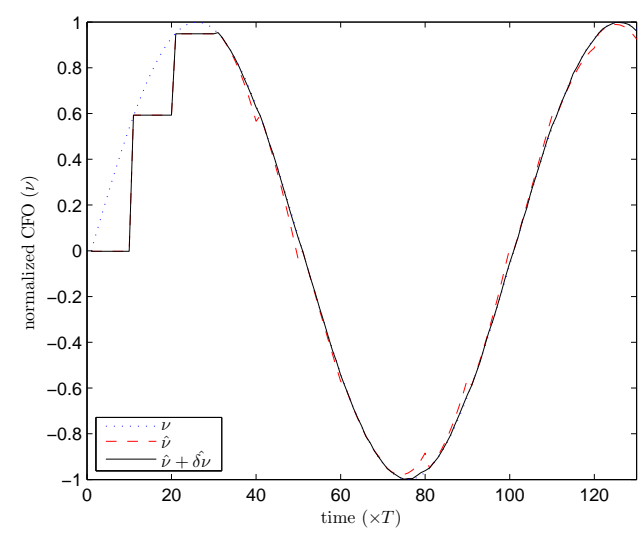

Fig.6: Estimates of the true normalized CFO (dotted line) obtained with the KF (dashed line) and the $K F$ combined with a decision-directed estimator (solid line). 

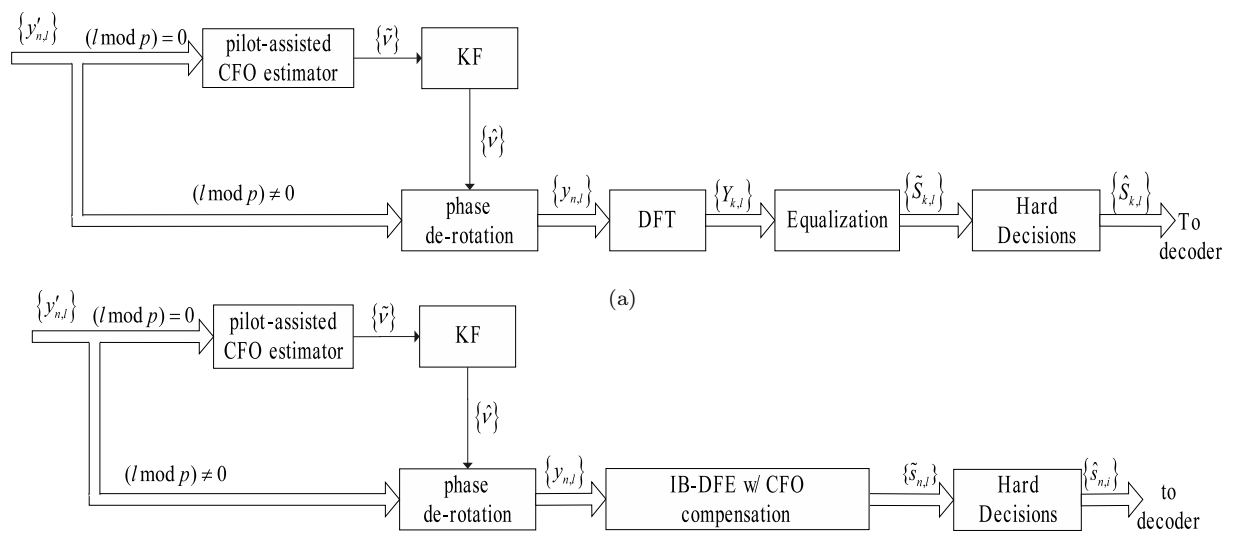

(b)

Fig.4: The proposed receiver:(a) for OFDM schemes and (b) for SC-FDE schemes.

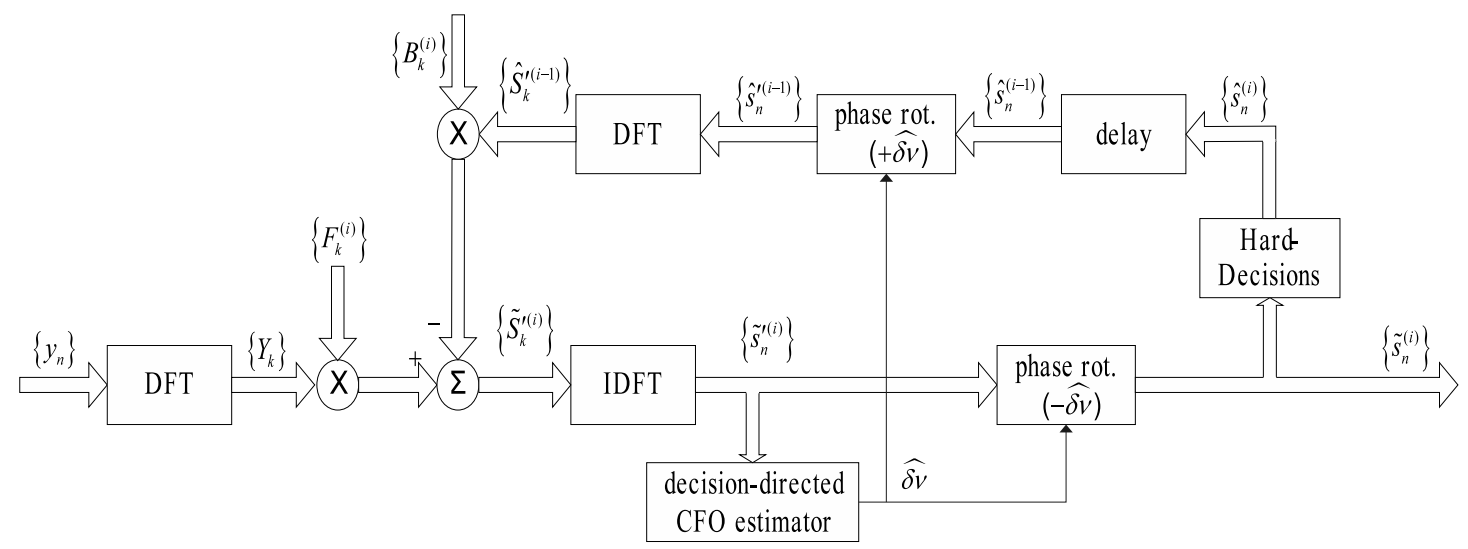

Fig.5: IB-DFE with CFO compensation.

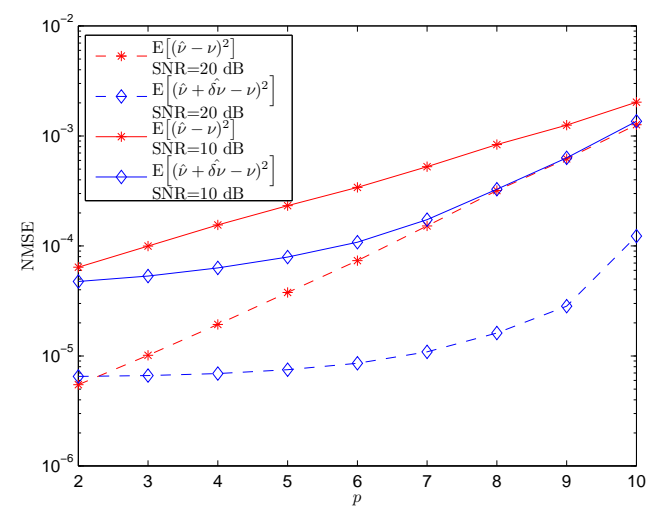

Fig.7: Normalized mean squared errors for the CFO estimation versus $p$.

modeled by a sinusoidal law

$$
v_{l}=v_{\max } \sin (2 \pi l / U), \quad l=0,1, \ldots
$$

where $v_{\max }$ is the maximum value of $v$ and $U$ is the period in symbol intervals.

The CFO that we assume present in our transmis- sions largely exceeds $1=(2 T)$. Under these conditions, conventional techniques for the CFO estimation are ineffective. This precludes the comparison between the proposed methods and non-recursive methods, since the BER rate performances for the latter are catastrophic. We refer to [7] for a comparison between our KF-based estimator with a non-recursive local estimator, regarding OFDM schemes.

Throughout this section we do $v_{\max }=1, U=100$ and divide the training symbols in $L=8$ sections.

We set the KF parameters to $q=10^{-3}$ and $r=$ 1. These parameters are slightly sub-optimal since the optimum values depend on the SNR. Notice that the amount of smoothing introduced by the KF can be controlled through the ratio $r / q$. For the pilotassisted CFO estimator we do $m=1$ and for decisiondirected CFO estimator we do $M_{d}=\lfloor 2 / 3 N\rfloor$, where $\lfloor x\rfloor$ means the larger integer that does not exceed $x$.

In Fig. 6 we plot the true $\mathrm{CFO}$, the estimates provided by the $\mathrm{KF}$ and the estimates obtained combining the $\mathrm{KF}$ with the decision-directed estimator for the time interval of 130 symbol intervals with SNR $=15 \mathrm{~dB}$ and $p=10$. We can see that the estimates 


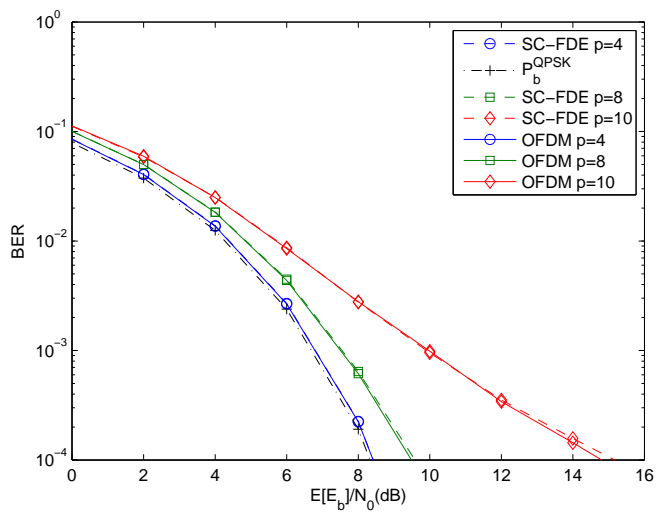

Fig.8: Uncoded BER performance of the proposed receivers for $A W G N$ channels. For comparison, we also plot the $M F B$.

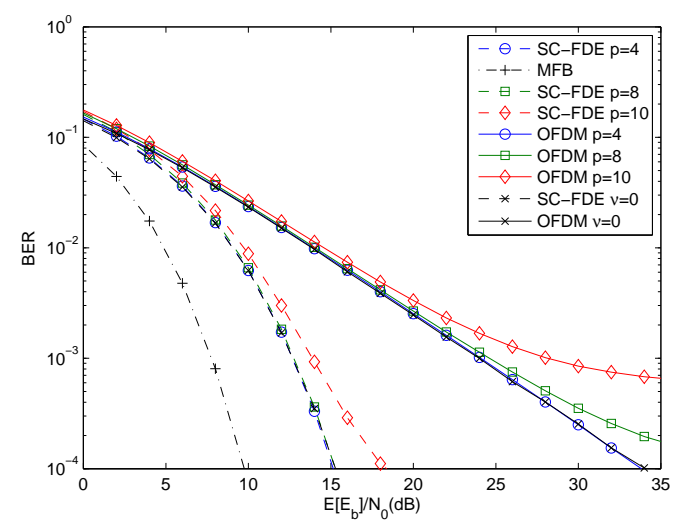

Fig.9: Uncoded BER performance of the proposed receivers. For comparison, we also depict the $S C$ $F D E$ and the OFDM performance with no CFO, i.e., $v=0$, and the $M F B$.

provided are able to track the true trajectory with only small errors. It is also visible that the combined use of a post-equalization decision-directed estimator provides increased accuracy. During the initial $3 p$ symbols intervals the estimates $\hat{x}_{2, n}$ and $\hat{x}_{4, n}$ are in convergence process and, thus, cannot be used for prediction. Instead, a piecewise constant CFO estimate is shown in that interval.

In Fig. 7 we present the CFO Normalized Mean Squared Errors (NMSE), $E\left\{[\hat{v}-v]^{2}\right\}$ and $E\left\{\left[\hat{v}+\hat{\delta}_{V}-v\right]^{2}\right\}$, of the estimates provided by the $\mathrm{KF}$ and the estimates obtained combining the $\mathrm{KF}$ with the decisiondirected estimator, respectively, versus the prediction step $p$ with $\mathrm{SNR}=10$ and 20 $\mathrm{dB}$. As expected the NMSE increases with the pilot symbol period. The combined use of the KF with the decision-directed estimator for the SC-FDE receiver yelds estimates with a NMSE, roughly, up to ten times smaller than the estimates provided solely by the KF.

Fig. 8 displays the uncoded BER performance of

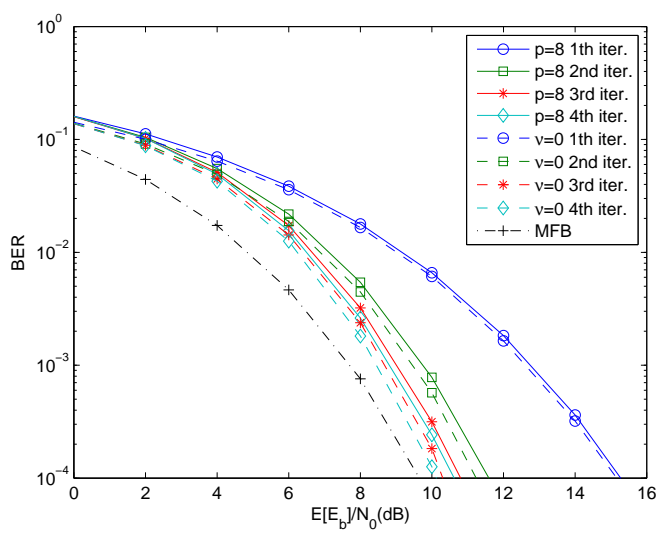

Fig.10: Uncoded BER performance for the different $I B-D F E$ iterations with pilot symbol period $p=8$. For comparison, we also depict the performance with no $C F O$, i.e., $v=0$, and the $M F B$.

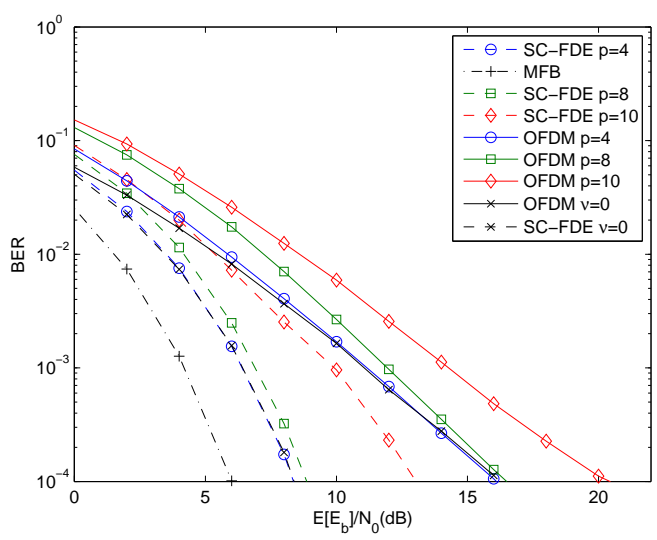

Fig.11: Uncoded BER performance of the proposed receivers with diversity order $J=2$. For comparison, we also depict the performance with no CFO, i.e., $\mathrm{v}$ $=0$, and the MFB.

the proposed receivers for AWGN channels. The following period symbols were used: $p=4 ; 8$, and 10 , which corresponds to $4 \%, 8 \%, 10 \%$ of the CFO repetition period $(U=100)$. Since the guard interval is much smaller than the useful symbol duration, we assume that the SNR loss due to discarding the cyclic prefix in the receiver is negligible. Both receivers have the same performance and for $p=4$ and 8 the curves are near the bit error probability for QPSK transmissions over AWGN channel (see the appendix for the expression).

In Fig. 9 we plot the uncoded BER performance, for the proposed receivers, in the presence of static multipath fading. For comparison, we display the BER performance curve of the SC-FDE and the OFDM schemes with no CFO, i.e., $v=0$. For $p$ $=4$ and 8 we see that, for both SC-FDE and OFDM schemes, the receivers performance is similar to that expected in a no CFO situation. With $p=10$ we see that for larger $\bar{B}_{b} / N_{o}$ there is an increasing performance degradation regarding the no CFO situation. 
It is important to note that this performance results are closely linked to the model chosen for the CFO variation. For larger pilot symbols periods, $p$, the CFO variations will be harder to track and, for increasing CFO variation rates, we will have increasing performance degradation. Comparing the proposed receivers performance for the current situation, we can see that the SC-FDE schemes presents significantly better results than that of OFDM. Furthermore, with the use of the IB-DFE, the SC-FDE BER performance can achieve results near the Matched Filter Bound (MFB) (see the appendix for the expression).

In Fig. 10 we can observe the improved BER performance obtained for the increasing iterations of the IB-DFE with CFO compensation. For $p=8$ we have for the 4 th iteration results near the MFB. For comparison, we also plot the SC-FDE performance with no CFO.

The uncoded BER performance of the receivers with two diversity branches, in the presence of static multipath fading, is displayed in Fig. 11. The use of diversity led to significant performance improvement in both schemes. Although OFDM schemes with $p=$ 4 and 8 have curves near to those obtained in a no CFO situation, we conclude that SC-FDE schemes display better results.

Fig. 12 depicts the coded BER performance of the proposed receivers in the presence of static multipath fading. We use a 64 states error correcting convolutional code with $1 / 2$ coding rate on both schemes. For comparison, the curves obtained in a no CFO situation are also represented. We observe improved performance on both receivers when compared with the uncoded transmission. Also, for the different values of $p$ the SC-FDE receiver have some gain over the OFDM.

\section{CONCLUSIONS}

We compared two receiver structures with $\mathrm{CFO}$ state-space estimation and compensation for both OFDM and SC-FDE schemes. For both schemes the $\mathrm{KF}$ offers accurate estimates of the rapidly-varying CFO. For the SC-FDE schemes, we provided an even more accurate $\mathrm{CFO}$ estimate by combining the $\mathrm{KF}$ with a post-equalization decision-directed CFO estimator. For the transmission scenarios over a static multipath fading channel simulated in this paper, the proposed receiver for SC-FDE exhibits better BER performance than that of OFDM. For AWGN channels the receivers perform equally.

Notice that, the used CFO model is not the best for the KF. In case the CFO has a good polynomial approximation the KF produces better estimates even if the CFO strongly varies between pilots, especially if the $\mathrm{KF}$ dimension is adequate.

\section{APPENDICES}

The bit error probability for QPSK transmissions over AWGN channel with Gray coding is given by [11]

$$
P_{b}^{Q P S K}(\gamma b)=Q\left(\sqrt{2 \gamma_{b}}\right)
$$

where $\gamma_{b}=E_{b} / N_{o}$ and $\mathrm{Q}$ is the normal distribution function

$$
Q(\mathrm{x})=\frac{1}{\sqrt{2 \pi}} \int_{\mathrm{x}}^{\infty} e^{-\frac{y^{2}}{2}} d y .
$$

The MFB for diversity scenarios is given by [10]

$$
P_{b}^{M F B}=E\left[P_{b}^{Q P S K}\left(\gamma_{b} \frac{1}{N} \sum_{j=1}^{J} \sum_{k=0}^{N-1}\left|H_{k}^{(j)}\right|^{2}\right)\right]
$$

where the expected value is computed over all $\left\{H_{k}^{(j)}\right\}$. The quantity

$$
\frac{1}{N} \sum_{j=1}^{J} \sum_{k=0}^{N-1}\left|H_{k}^{(j)}\right|^{2}
$$

measures the fading for each channel realization, so (32) is the best performance that we can have if no ISI exists, in this specific case for the transmission of QPSK symbols with Gray coding.

\section{References}

[1] L. Cimini Jr., Analysis and simulation of a digital mobile channel using orthogonal frequency division multiplexing, IEEE Trans. Commun., vol. 33, pp. 400411, Jul. 1985.

[2] H. Sari, G. Karam, and I. Jeanclaude, An analysis of orthogonal frequency-division multiplexing for mobile radio applications, in Proc. IEEE Vehicular Technology Conference, (VTC94), Stockholm, Jun. 1994, pp. 16351639.

[3] H. Meyer, M. Moeneclaey, and S. Fetchel, Digital Communication Receivers, 2nd ed. New York: Wiley, 1998.

[4] P. Moose, A technique for orthogonal frequency division multiplexing frequency correction, IEEE Trans. Commun., vol. 42, pp. 2908 2914, Oct. 1994.

[5] T. Schmidl and D. Cox, Robust frequency and timing synchronization for OFDM, IEEE Trans. Commun., vol. 45, pp. 16131621, Dec. 1997.

[6] M. Morelli and U. Mengali, An improved frequency offset estimator for OFDM applications, IEEE Commun. Lett., vol. 3, pp. 7577, Mar. 1999.

[7] F. Nunes and J. Leito, State-space estimation of rapidly-varying carrier frequency offsets in OFDM 
systems, in Proc. IEEE Signal Processing Advances in Wireless Communications (SPAWC03), Rome, Jun. 2003.

[8] M. Alard and L. Lassalle, Principles of modulation and channel coding for digital broadcasting for mobile receivers, EBU Technical Review, no. 224, pp. 168190, Aug. 1987.

[9] T. Arajo and R. Dinis, Iterative equalization and carrier synchronization for single-carrier transmission over severe time-dispersive channels, in Proc. IEEE Global Communicatons Conference (GLOBECOM04), vol. 5, 29 Nov. - 3 Dec. 2004, pp. 31033107.

[10] R. Dinis, A. Gusmo, and N. Esteves, On broadband block transmission over strongly frequencyselective fading channels, in IEEE Wireless03, 2003.

[11] J. Proakis, Digital Communications, 4th ed. New York: McGraw-Hill, 2001.

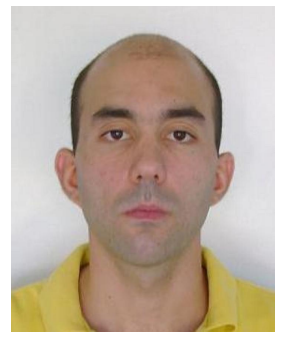

Pedro Pedrosa received the M.Sc. degree in electrical and computer engineering from Instituto Superior Tecnico (IST), Lisbon, Portugal, in 2007, where he is currently pursuing the Ph.D. degree, also in electrical and computer engineering. His current research interests include detection, estimation and prediction of the carrier frequency offset for block transmissions with frequencydomain equalization.

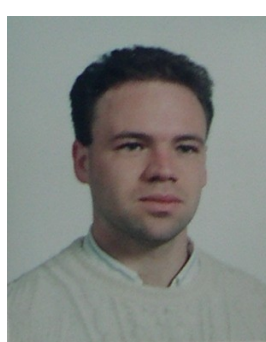

Rui Dinis received the Ph.D. degree from Instituto Superior Tcnico (IST), Technical University of Lisbon, Portugal, in 2001. Since 2001 he has been a Professor at IST. He was a member of the research center CAPS/IST (Centro de Anlise e Processamento de Sinais) from 1992 to 2005 . Since 2005 he is a member of the research center ISR/IST (Instituto de Sistemas e Robtica). He has been involved in several research projects in the broadband wireless communications area. His main research interests include modulation, equalization and channel coding.

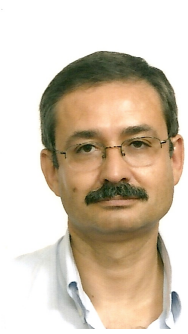

Fernando D. Nunes received his E.E., M.Sc. and Ph.D. degrees in Electrical Engineering all from the Instituto $\mathrm{Su}-$ perior Tcnico (IST), Technical University of Lisbon, Portugal. He is currently an assistant professor at the Department of Electrical and Computer Engineering, IST, and researcher with the Communication Theory and Pattern Recognition Group at the Instituto de Telecomunicaes in Lisbon. His current research interests include communication theory and signal processing in mobile radio communications and navigation systems. 